\section{Avalanche Triage: Are Two Birds in the Bush Better Than One in the Hand?}

To the Editor:

In a previous issue of Wilderness and Environmental Medicine, Bogle, Boyd, and McLaughlin discuss a proposed triage algorithm for use in avalanche incidents in which the number of victims exceeds the capacity of the rescuers to give optimum treatment to each victim. ${ }^{1}$ They claim that this algorithm, which they call "AvSORT," will help rescuers "rapidly identify those that will benefit from limited resources."

While a triage algorithm for multiple casualty avalanche incidents might be useful, the proposed algorithm has potential problems. The main difficulty is the assumption that someone who is still buried might have a better chance of suvival than someone who is extricated without obviously lethal injuries. This assumption is contrary to the common wisdom that a bird in the hand is worth two in the bush.

The authors quote statistics showing that there have been many fatalities in avalanches with multiple victims, but they have not analyzed past avalanche incidents to see what effects their proposed algorithm might have had if it had been applied in actual incidents. Instead they have modified existing trauma triage algorithms in a data vacuum. While they claim that the AvSORT avalanche would produce desirable percentages of overtriage and undertriage, they present no evidence in support of this assertion. They also present no evidence that their algorithm would result in an increased number of survivors.

The authors are not specific about the conditions under which their proposed algorithm should be used. What is the definition of a "mass casualty incident"? For example, does this term apply in the case of 2 avalanche victims and 2 uninjured rescuers? The algorithm also does not define how first responders should determine when they are "overwhelmed," nor does it give criteria for determining if the area is "remote." Is an area remote if the response time for rescue is 2 hours? Arrested hypothermic patients have been successfully rewarmed after 4 hours of cardiopulmonary resuscitation (CPR), even in Europe. ${ }^{2}$

It is important that the proposed algorithm not be used as a reason to deviate from established resuscitation guidelines except under the most extenuating circumstances. Apneic, severely hypothermic avalanche victims have been successfully resuscitated. ${ }^{2}$ It is critical to recognize that victims buried for more than 35 minutes who have a patent airway have a chance of survival. The guiding principle should be not to give up! It would be tragic if a rescuer did not try to resuscitate such a patient due to feeling "overwhelmed." The proposed algorithm should be modified so that victims buried longer than 35 minutes who have a patent airway but are not breathing not be triaged to the "expectant" category. They should receive CPR and be transferred to a hospital capable of performing cardiopulmonary bypass.

Because the authors are advocating a triage guideline, they have not specified all the specifics of treatment in the various triage categories. For this, rescuers will need to follow another algorithm, such as the International Commission for Mountain Emergency Medicine (ICAR MEDCOM) avalanche resuscitation guidelines. ${ }^{3}$ The authors present these guidelines but state that they "rarely" apply to first responders in North America. It is true that the ICAR MEDCOM guidelines cannot be fully applied without the use of cardiac monitoring, but they are predominantly based on clinical criteria that do not require special equipment. The authors note that if the number of rescuers is adequate to search for buried victims as well as treat extricated victims, standard treatment guidelines apply. In this case, there is no need for a triage algorithm.

Although the proposed algorithm makes use of the duration of burial to determine treatment, it does not use duration as a prognostic factor for buried victims. Avalanche survival probability of buried victims is $91 \%$ at 18 minutes but drops precipitously to $34 \%$ after 35 minutes as victims without an air pocket die from asphyxiation. ${ }^{4}$ Survival then decreases gradually to a mere $7 \%$ at 130 minutes as victims with a "closed" air pocket succumb to slow asphyxia and hypothermia. It makes less sense to divert resources from treating extricated victims with a chance of survival to searching for buried victims after about 2 hours than it does at 35 minutes. Unfortunately, the reality of avalanche incidents, even in Europe, is that increased rescue capabilities have not increased survival. This is because the best chance for an avalanche victim to survive is to be extricated rapidly by other members of the party who were not buried.

Another problem with the proposed algorithm is the designation of destination hospital. While the European Alps are dotted with hospitals only a 15-minute helicopter flight from almost any point and referral centers capable of intensive care within 30 minutes, the situation in North America is that even the closest hospital may be an hour or more away by helicopter and "trauma centers" are often beyond the range of helicopter transport at all. In the case of apneic hypothermic patients, the most appropriate destination hospital may not be a trauma center but should be a center capable of performing cardiopulmonary bypass.

A recent case report ${ }^{5}$ illustrates the major potential pitfall of the proposed triage algorithm. In this case report, 2 skiers were completely buried in an avalanche in the Italian Alps. Uninjured companions activated an emergency response by cell phone. Amazingly, the first 
victim was breathing when extricated from a depth of $3 \mathrm{~m}$ (9.8 ft) after 100 minutes. However, he was unconscious and severely hypothermic with an epitympanic core temperature of $22^{\circ} \mathrm{C}\left(71.6^{\circ} \mathrm{F}\right)$. The second skier was found a few minutes later in asystole without an air pocket. He was declared dead by the emergency physicians on site, according to the ICAR avalanche guidelines.

The first patient was intubated and transported to the nearest hospital by helicopter. During transport he went into ventricular fibrillation. He continued to be ventilated, but chest compressions were not administered for 15 minutes. Initial attempts at defibrillation at the destination hospital were unsuccessful because of hypothermia. He was subsequently transferred to a referral hospital where he could be placed on cardiopulmonary bypass. He was successfully resuscitated and rewarmed. He eventually made a complete neurologic recovery.

Had there been few rescuers rather than the many who actually responded and had the AvSORT algorithm been applied, the first victim would have been triaged to the "immediate treatment" category and transferred to a trauma center. However, if ventricular fibrillation had started before he was placed in the helicopter and the second victim had not yet been found, the first victim would have been triaged or retriaged into the category of "expectant" management and would have received no further treatment. The result would have been a fatal outcome rather than the complete recovery he actually experienced.

Until the authors of the AvSORT algorithm can produce convincing data to show that their proposed triage method would increase survival in multi-casualty avalanche incidents, rescuers should resuscitate potentially live victims who have already been found and not divert necessary medical resources to further searching.

Ken Zafren, MD

Alaska Mountain Rescue Group, International Commission for Mountain Emergency Medicine (ICAR MEDCOM), Alaska Native Medical Center, Anchorage, AK; Stanford University Medical Center, Stanford, CA, USA

\section{References}

1. Bogle LB, Boyd J, McLaughlin KA, Triaging multiple victims in an avalanche setting: the Avalanche Survival Optimizing Rescue Triage (AvSORT) algorithmic approach. Wilderness Environ Med. 2010;21:28-34.

2. Althaus U, Aeberhard P, Schüpbach P, Nachbur BH, Mühlemann W. Management of profound accidental hypothermia with cardiorespiratory arrest. Ann Surg. 1982;195:492-495.
3. Brugger H, Durrer B. On-site treatment of avalanche victims ICAR-MEDCOM-recommendation. High Alt Med Biol. 2002;3:421-425.

4. Falk M, Brugger H, Adler-Kastner L. Avalanche survival chances. Nature. 1994;368:21.

5. Oberhammer R, Beikircher W, Hörmann C, et al. Full recovery of an avalanche victim with profound hypothermia and prolonged cardiac arrest treated by extracorporeal rewarming. Resuscitation. 2008;76:474-480.

\section{In Reply to Avalanche Triage}

\section{To the Editor:}

In reply to the letter by Dr Ken Zafren, we take this opportunity to resolve any confusion with the use of the Avalanche Survival Optimizing Rescue Triage (AvSORT) algorithm. ${ }^{1}$

On February 1, 2003 in backcountry Canada, 2 mountain guides watch with horror as an avalanche engulfs and buries a school group of 17 participants below them. ${ }^{2-4}$ They immediately realize their priority for rescue is to uncover as many victims as possible prior to the onset of asphyxia. They "cannot save everybody" 4 but must focus on "the greatest good for the greatest number." ${ }^{\text {-11 }}$ They decide, as 2 rescuers, they will only dig enough to allow resumption of breathing, to clear airways, and to hand responsive victims their own shovels for self-extrication before moving on. They recognize that to stop and attempt cardiopulmonary resuscitation (CPR) on any one asphyxiated victim will seriously compromise the survival of other victims still buried. The first victim, recovered in the first 5 minutes, is the school teacher who calls on his satellite phone for outside organized rescue. As the avalanche debris sets up "like concrete," victims who are not buried too deep are uncovered while deep burials are passed over due to the pressure of time. One victim goes on, after fully extricating himself, to locate and uncover 3 of his classmates, resulting in their survival. The first rescue helicopter lands after 55 minutes and of the 17 buried victims 10 survive. The rational action of the 2 mountain guides is credited with the survival of the majority of these victims in this mass casualty avalanche incident. ${ }^{3,4}$ The development of our proposed AvSORT algorithm is a result of this and other similar incidents that we cite in the text of our article. This algorithm is designed for the "initial management of mass casualty avalanche incidents when manpower is overwhelmed." We must re-emphasize this.

By comparison Dr Zafren cites an incident in which 3 helicopters with 15 rescuers, 2 emergency physicians, and 2 dog handlers are on scene for 2 buried victims, 1 of whom survives. ${ }^{12}$ This would never be considered a 